\title{
Comparing LIMERIC and DCC approaches for VANET Channel Congestion Control
}

\author{
Gaurav Bansal $^{\dagger}$, Bin Cheng ${ }^{\ddagger}$, Ali Rostami ${ }^{\ddagger}$, Katrin Sjoberg ${ }^{6}$, John B. Kenney ${ }^{\dagger}$, and Marco Gruteser ${ }^{\ddagger}$ \\ †Toyota InfoTechnology Center, USA \\ ${ }^{\ddagger}$ Rutgers University, USA \\ ${ }^{6}$ Volvo Groups Trucks Technology, Sweden \\ Email: gbansal@us.toyota-itc.com, cb3974@winlab.rutgers.edu, a.rostami@ rutgers.edu, katrin.sjoberg@volvo.com, \\ jkenney@us.toyota-itc.com, gruteser@winlab.rutgers.edu
}

\begin{abstract}
Channel congestion is one of the major challenges for IEEE 802.11p-based vehicular ad hoc networks. Unless controlled, congestion increases with vehicle density, leading to high packet loss and degraded safety application performance. In this paper, we study two classes of congestion control - reactive and adaptive. The reactive approach is represented by the Decentralized Congestion Control (DCC) framework defined in ETSI. The adaptive approach is represented by the LIMERIC linear control algorithm. Both approaches control safety message transmission as a function of channel load (i.e. Channel Busy Ratio, CBR). A reactive approach uses CBR directly, defining an appropriate transmission behavior for each CBR value, e.g. via a table lookup. By contrast, an adaptive approach identifies the transmission behavior that drives CBR to a target channel load, thus achieving the best message throughput possible for any given vehicle density. The paper considers two variations of DCC, one in which it serves as a traffic shaping "gatekeeper" above the MAC sublayer, and another in which it additionally limits safety message generation at the facilities layer. The paper has two main results. First, it is shown that LIMERIC generally outperforms both DCC variations in a winding road scenario with various vehicle densities. Inter-packet reception gap and position tracking error are the primary metrics. This advantage is due to primarily LIMERIC's ability to achieve a target load consistent with maximum throughput and vehicle awareness. Second, it is shown that both DCC variations are subject to steady state oscillations, and the case in which DCC also limits message generation is subject to truly unstable variations. The paper uses NS-2 simulation results to support these conclusions.
\end{abstract} DSRC

Keywords-DCC, congestion control, IEEE 802.11p, ITS-G5,

\section{INTRODUCTION}

Cooperative Intelligent Transport System (C-ITS) technology enables a wide variety of vehicular ad hoc networking applications, including collision avoidance, road hazard awareness, and route guidance. Based on the Medium Access Control (MAC) and Physical Layer (PHY) protocols specified in the IEEE 802.11p standard [3], C-ITS is moving rapidly towards deployment in Europe and other regions. Twelve members of the Car-2-Car Communications Consortium (C2C-CC) have mutually pledged to begin equipping their vehicles with C-ITS by 2015 [4]. In the US, where the technology is known as Dedicated Short Range Communication (DSRC), the deployment landscape was recently clarified when the government announced an intention to require this equipment in new cars within a few years [5].

One area of active C-ITS/DSRC research is channel congestion control. With a typical range of hundreds of meters, a C-ITS device may share a $10 \mathrm{MHz}$ channel with hundreds or even a few thousand other devices. The Carrier Sense Multiple Access/Collision Avoidance (CSMA/CA) MAC protocol used in C-ITS is optimized for low-to-moderate channel loads. With increasing load, the channel becomes saturated, the probability of overlapping transmissions (i.e. packet collisions) approaches one, and the aggregate channel throughput falls off after reaching a plateau.

While in general a C-ITS channel may support a variety of applications, congestion in the $5.9 \mathrm{GHz}$ spectrum is likely to be associated with a high volume of vehicle safety messages. These are Cooperative Awareness Messages (CAMs) [6] in Europe and Basic Safety Messages (BSMs) in the US [7]. Congestion reduces the rate at which these safety messages are successfully communicated to neighbors, and the resulting reduced awareness harms the C-ITS safety mission. The focus of this paper is on controlling congestion by controlling these messages. The results are readily extended to include nonsafety messages. We explore the CAM (or BSM) transmission rate as the primary means of control, though the approaches can also be applied to power or more complex hybrid controls.

One of the challenges of C-ITS congestion control is the inherent cross-layer nature of the problem. For example, Channel Busy Ratio (CBR) is a PHY measure of congestion, MAC traffic shaping may be necessary to account for multiple protocols at higher layers (e.g. the addition of IP traffic to CAMs), the network layer may be invoked to disseminate congestion information, and CAMs are actually generated at the facilities layer. In this paper we investigate two rate control points in the stack: a congestion-sensitive traffic shaping function at the MAC, or CAM generation at the facilities layer, as well as a combination of both.

The primary contribution of this paper is a comparison between two general approaches to C-ITS congestion control: reactive and adaptive. Both use $\mathrm{CBR}$ as an input. In the reactive approach, represented by the ETSI DCC framework [8], control variables (e.g. message rate) are direct functions of CBR. One example of a reactive approach is a table lookup with CBR as input and message rate as output. In the adaptive 
approach, represented by the LIMERIC algorithm [1], the measured CBR is compared to a target CBR, and the algorithm adapts control variables to drive CBR toward the target. The paper has two principal results. First, the LIMERIC adaptive approach generally achieves lower packet reception intervals and tracking error than DCC, due to its ability to optimize throughput. Second, the DCC approach tends to steady state oscillations, due to discrete states, and more importantly is shown to exhibit true instability for the case where DCC controls both facilities layer message generation and MAC layer shaping.

The algorithm comparison is carried out via NS-2 simulations of a winding road scenario previously utilized in [2]. Simulation results for four approaches are shown: no control, LIMERIC, DCC acting as a traffic shaping gatekeeper at the access layer, and DCC limiting message generation at facilities layer. Three vehicle densities are tested. The primary performance metrics are reception interval and tracking error.

Following this introduction, Section 2 describes the usage and generation rules for the CAM and BSM. Section 3 explains the DCC and LIMERIC approaches. Simulation results comparing these approaches are presented in Section 4, and conclusions are presented in Section 5.

\section{PROTOCOL STACK FOR C-ITS}

The European C-ITS architecture was ratified by ETSI in September 2010. It is depicted in Fig. 1. Here the PHY and data link layer have been grouped into an access layer. The OSI transport and network layers are considered as a single "networking \& transport" entity. The highest layer in the architecture, providing services to the applications, is referred to as the facilities layer, a name that reflects the influence of not only communication protocols, but also of exogenous inputs such as Global Navigation Satellite System (GNSS) and in-vehicle sensor data. Fig. 1 also contains a comparison between the European protocol stack and the Wireless Access in Vehicular Environment (WAVE) architecture used in the US. The major differences are: ( $i)$ in Europe, DCC is required by regulation (EN 302571 [9]) and it must be situated at the access layer, whereas there is not yet a DCC regulation in the US; (ii) at the networking \& transport layer, Europe has support for multihop communication through GeoNetworking (GeoNet), whereas no such capability is specified in the US; and (iii) in the US events like "hard braking" are indicated within the BSM, while in Europe such events are communicated not by the CAM but rather in a distinct message type, the Decentralized Environmental Notification Message (DENM). The common elements between US and Europe are IEEE 802.11p and LLC at the lower layers. In addition, a high degree of harmonization has been achieved between the BSM and CAM.

\section{A. BSM and CAM generation}

The position messages, BSM and CAM, will be the basis for increased road traffic safety. They contain more or less the same information with some regional differences. The major difference concerns how they are generated. The BSM structure is outlined in SAE J2735 [7] and CAM in EN 302 637-2 [6], and they contain position information, time stamp, heading, speed, driving direction, path history, vehicle type etc.

\begin{tabular}{|c|c|c|c|c|}
\hline osı layers & WAVE & \multicolumn{3}{|c|}{ ETSI TC ITS architecture } \\
\hline Application & SAE BSM & CAM & DENM & Facilities \\
\hline Transport & \multirow{2}{*}{$\begin{array}{l}\text { IEEE } \\
1609.3\end{array}$} & \multicolumn{2}{|c|}{ BTP } & \multirow{2}{*}{$\begin{array}{l}\text { Networking \& } \\
\text { transport }\end{array}$} \\
\hline Network & & Geo & Net & \\
\hline \multirow{3}{*}{ Data link } & \multicolumn{3}{|c|}{ LLC } & \multirow{4}{*}{ Access } \\
\hline & IEEE1609.4 & DC & $\mathrm{CC}$ & \\
\hline & \multicolumn{3}{|c|}{ IEEE $802.11 \mathrm{p}$} & \\
\hline Physical & \multicolumn{3}{|c|}{ IEEE $802.11 p$} & \\
\hline
\end{tabular}

Figure 1. Comparison of the protocol stacks in the US, called "WAVE," and the EU stack developed by ETSI TC ITS.

CAMs are generated at intervals of no less than $100 \mathrm{msec}$ and no more than $1000 \mathrm{msec}$, with specific generation times determined by DCC and vehicle dynamics. CAM generation rules are complex. Generally, a new CAM shall be generated when both of the following conditions, measured relative to the prior CAM message, are met: the interval provided by DCC, via the T_GenCam_DCC parameter, expires, and one of the following dynamics criteria are met: $(i)$ heading changed $>4^{\circ}$, (ii) position changed $>4$ meters, or (iii) magnitude of speed changed $>0.5 \mathrm{~m} / \mathrm{sec}$. A CAM is also generated after one second even if the two conditions are not met. When a CAM is triggered by one of the dynamics conditions, a second and third CAM will also be generated at the same intervals unless subsequent dynamics lead to an even shorter interval. T_GenCam_Dcc is set via the management plane by the congestion control mechanism (DCC) residing in the access layer.

BSM generation rules have not yet been specified in the US. Most testing and trials have used a fixed $10 \mathrm{BSM} / \mathrm{second}$ rate. Specific generation rules will most likely be standardized as part of a congestion control algorithm for precise channel access control.

\section{CONGESTION CONTROL}

Congestion control is a function that modifies transmission behavior in response to network load to increase communication reliability. CAMs and BSMs are broadcasted in ad hoc networks, rendering traditional automatic repeat request (ARQ) feedback infeasible. The best feedback in IEEE 802.11p networks is CBR. If congestion control is not present, the channel can be overloaded as the vehicle density increases. Congestion control improves predictability, reliability and efficient use of channel resources, and is considered a necessary function in vehicular networks. Common methods for congestion control are: (i) transmit message rate control (TRC), (ii) transmit power control (TPC), and (iii) transmit data rate control (TDC). The focus in this paper is on TRC. 
We now present two different approaches for congestion control: a reactive approach, represented by the European DCC framework, and an adaptive approach represented by the LIMERIC algorithm.

\section{A. Reactive control: European DCC}

TS 102687 [8] outlines a DCC framework for Europe. Conformance to TS 102687 is a requirement in the harmonized EN 302571 [9], regulating the European C-ITS frequency bands. TS 102687 is a toolbox, with several optional methods. The most prominent method is a table lookup using TRC. In this paper, we have parameterized this table look-up; see Table 1, where the packet transmission rate is specified as a function of measured CBR. The specific values in Table 1 are consistent with those under consideration for trials and deployment.

Table 1. Table look-up for congestion control.

\begin{tabular}{|c|c|c|}
\hline Channel load & Packet Tx interval & Packet rate \\
\hline$<30 \%$ & $100 \mathrm{~ms}$ & $10 \mathrm{~Hz}$ \\
\hline $30-39 \%$ & $200 \mathrm{~ms}$ & $5 \mathrm{~Hz}$ \\
\hline $40-49 \%$ & $300 \mathrm{~ms}$ & $3.33 \mathrm{~Hz}$ \\
\hline $50-59 \%$ & $400 \mathrm{~ms}$ & $2.5 \mathrm{~Hz}$ \\
\hline$>59 \%$ & $500 \mathrm{~ms}$ & $2 \mathrm{~Hz}$ \\
\hline
\end{tabular}

It is evident from Table 1 that there is no control above a channel load of $59 \%$, leaving only the MAC protocol to manage channel usage as the CBR increases. DCC in Europe currently limits CAM generation in the facilities layer and imposes a gatekeeping (traffic shaping) function just above the MAC layer. DCC produces an interval, T_GenCam_Dcc, which limits CAM generation in the facilities layer, and also shapes traffic into the MAC layer. In this paper, if a CAM is generated before the prior CAM is passed to the MAC, the prior CAM is replaced by the new one, such that no more than one CAM is in the gatekeeping queue at a time. In Europe, event-based (DENM) messages may also be generated, but these are not included in the simulations of this paper.

\section{B. Adaptive control: LIMERIC}

LIMERIC [1] is a distributed and adaptive linear ratecontrol algorithm where each vehicle adapts its message rate in a way such that the total channel load converges to a specified target. The message rate for the $j^{\text {th }}$ vehicle (denoted as $r_{j}$ ) is adapted according to the following equation:

$$
r_{j}(t)=(1-\alpha) r_{j}(t-1)+\beta\left(r_{g}-r_{C}(t-1)\right)
$$

where $r_{C}$ is the total rate of all $K$ vehicles in a given area, $r_{g}$ is the target for total rate, and $\alpha$ and $\beta$ are adaptation parameters that control stability, fairness, and steady state convergence. It is shown using linear systems theory that in steady state LIMERIC converges to a unique and fair rate for all vehicles, and the total rate $r_{C}$ converges to $r_{f}$ which is proven as [1]:

$$
r_{f}=\frac{K \beta r_{g}}{\alpha+K \beta}
$$

Stability conditions and convergence speed are also derived, and it is shown that LIMERIC adapts quickly to changing network conditions. For a practical implementation of
LIMERIC, the CBR created from all $K$ vehicles is used to estimate the total rate $r_{C}(\mathrm{t})$, and the target channel load $r_{g}$ is then mapped to an equivalent CBR. CBR is measured every $\delta$ time and the rate is adapted according to equation (1). More details are provided in [1].

\section{NUMERICAL RESULTS}

In this Section, the performance of various congestion control algorithm discussed in the paper will be evaluated with ns2 simulations. As metrics we use the Packet Error Rate (PER), the $95^{\text {th }}$ percentile Inter-Packet Gap (IPG) and the Tracking Error (TE). TE is defined as the error between the transmitter's true location and the receiver's perception of the transmitter's location. The receiver extrapolates the transmitter's location using the GPS information in the most recently received message and using a constant-speed constantheading coasting model. The IPG and the TE are related, but the IPG measures the time between two consecutive received packets and is an important metric since it characterizes how fresh the received information from other vehicles is. While the $\mathrm{TE}$ is an application-oriented measure of how accurately a receiving vehicle can track the movements of a sending vehicle.

We compare the performance results of the LIMERIC algorithm, fixed $10 \mathrm{~Hz}$ transmissions (no congestion control present), CAM generation implemented with European DCC approach (messages are generated according to vehicle dynamics as described in Section II and adhere to the T_GenCam_Dcc parameter set by the access layer), and fixed $10 \mathrm{~Hz}$ generation implemented with European DCC approach (messages are generated at a fixed $10 \mathrm{~Hz}$ rate and not affected by vehicle dynamics; DCC acts as a gatekeeper in the access layer). The traffic models for the four approaches are detailed in Table 2.

Table 2. Data traffic models used in the simulations.

\begin{tabular}{|c|l|}
\hline Name & \multicolumn{1}{c|}{ Description } \\
\hline $10 \mathrm{~Hz}$ & $\begin{array}{l}\text { There is no congestion control algorithm present } \\
\text { and all vehicles transmit CAM/BSM 10 times per } \\
\text { second. }\end{array}$ \\
\hline LIMERIC & $\begin{array}{l}\text { The vehicles generate and transmit CAM/BSMs } \\
\text { when LIMERIC algorithm allows. }\end{array}$ \\
\hline 10Hz with DCC & $\begin{array}{l}\text { The vehicles generate CAM/BSM with 10 Hz and at } \\
\text { the access layer the DCC will act as a gatekeeper } \\
\text { according to the current allowed rate specified in } \\
\text { Table 1. }\end{array}$ \\
\hline CAM with DCC & $\begin{array}{l}\text { The vehicles generate CAMs according to EN } \\
\text { 302 637-2 (also described in Section II), which is } \\
\text { based on vehicle dynamics and CAMs are } \\
\text { generated when the T_GenCam_Dcc parameter } \\
\text { allows. }\end{array}$ \\
\hline
\end{tabular}

\section{A. Simulation configurations}

The SUMO mobility simulator has been used to configure a highway of length $4 \mathrm{~km}$, with 3 lanes in each direction. The middle part of the road is a winding section of length $375 \mathrm{~m}$ (with the radius of the winding part set to be $40 \mathrm{~m}$ ), see Fig. 2. This configuration permits testing of the performance of the algorithms (European DCC, LIMERIC) not only on a straight part of the road where vehicles have relatively low dynamics but also on the winding part of the road where vehicles experience high dynamics. This is important since the CAM 
generation depends on vehicle dynamics. The average desired speed of the vehicles on the three lanes on the highway were $19 \mathrm{~m} / \mathrm{s}$ in the fastest lane (left lane), $18 \mathrm{~m} / \mathrm{s}$ in the middle lane and $17 \mathrm{~m} / \mathrm{s}$ in the slowest lane (right lane).

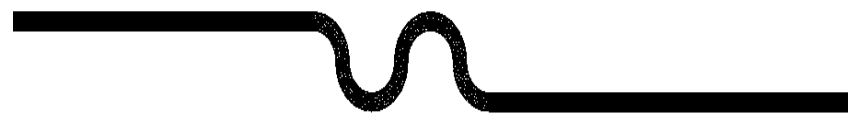

Figure 2. Road topology for simulations as in [2].

NS 2.34 has been used to run the simulations for three density scenarios with 500, 1000 and 1500 vehicles, respectively, on the $4 \mathrm{~km}$ road. The wireless channel propagation is Nakagami distributed, with the same parameters as in [2]. The list of simulation parameters used in this work is given in Table 3 (also, note that the parameters for CAM generation and European DCC are used as specified in Section II, III):

Table 3. Simulation parameters.

\begin{tabular}{|c|c|}
\hline Common Parameter & Value \\
\hline Noise floor & $-99 \mathrm{dBm}$ \\
\hline Carrier sense threshold & $-96 \mathrm{dBm}$ \\
\hline $\begin{array}{c}\text { Packet reception SINR (for 6 Mbps } \\
\text { packet) }\end{array}$ & $7 \mathrm{dBm}$ \\
\hline Payload size & $350 \mathrm{bytes}$ \\
\hline Transmission rate & $6 \mathrm{Mbps}$ \\
\hline Transmission power & $\begin{array}{c}10 \mathrm{dBm} \text { (corresponds to } \\
\text { theoretical range of } 500 \mathrm{~m} \text { in } \\
\text { our configuration of NS-2) }\end{array}$ \\
\hline GPS update frequency & $10 \mathrm{~Hz}$ \\
\hline CBR measurement period & $100 \mathrm{msec}$ \\
\hline LIMERIC & $200 \mathrm{msec}$ \\
\hline$\delta$ & .68 \\
\hline Goal CBR convergence & 0.033 \\
\hline A & 0.1 \\
\hline CAM Generation & $10 \mathrm{msec}$ \\
\hline CAM generation checking period &
\end{tabular}

Note, that all the performance metrics in the paper are calculated on the winding part of the road where vehicles experience high dynamics. For the results in Section IV.B, we have synchronized the CBR measurement period across all nodes, i.e. each node measures the CBR at its own location, but all nodes measure $\mathrm{CBR}$ at the same time. Synchronization using GPS is known to be quite accurate. In Section IV.C, we also provide results for the case when CBR measurements are asynchronous.

\section{B. Numerical Results}

In Figure 3 - Figure 5, the PER comparison for the four different data traffic models (Table 2) for the three different vehicle densities are depicted. We observe that CAM with DCC has much higher PER than LIMERIC and $10 \mathrm{~Hz}$ with DCC for all vehicle densities. The high PER for CAM with DCC, stems from very fluctuating CBR measurements. In Figure 13, a sixty second snapshot on CBR measurements for the different schemes are depicted for the 1000 nodes case. It is clearly seen that the CBR measurements for CAM with DCC suffers from strong oscillations.

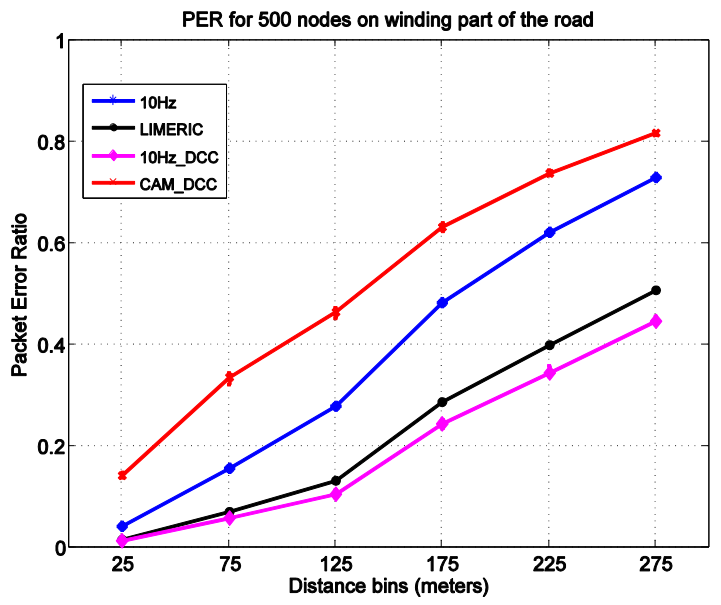

Figure 3. PER for total number of vehicles $=\mathbf{5 0 0}$.

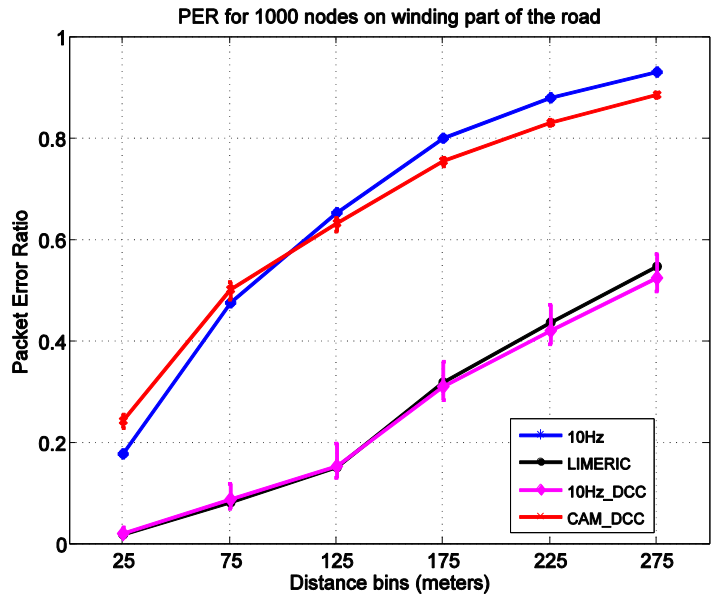

Figure 4. PER for total number of nodes $=1000$.

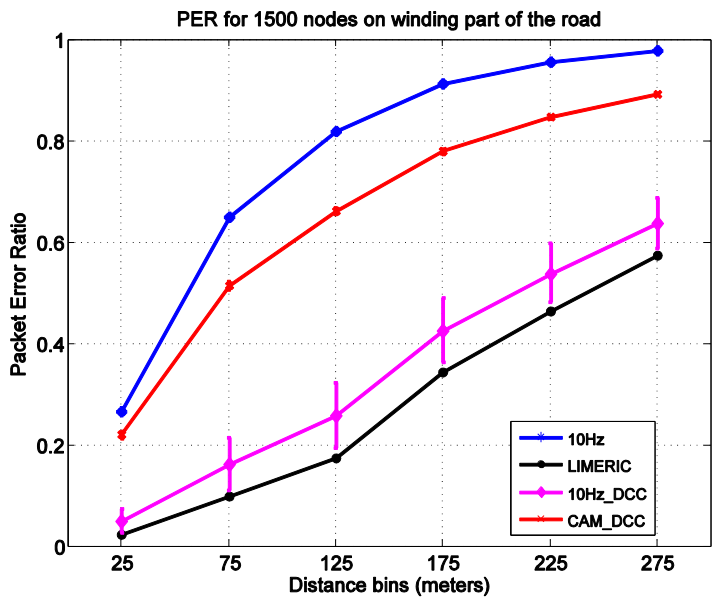

Figure 5. PER for total number of nodes $=1500$

According to the CAM generation rules, the oscillation in the CBR also leads to the oscillation in the message generation 
times. The vehicles that are close to each other measure similar $\mathrm{CBR}$ values at the end of a CBR measurement interval and then generate messages at similar intervals. This leads to the synchronized message transmissions from neighboring vehicles and it results in a higher PER even when the average CBR value is low.

In Figure 6, the number of transmissions occurring in a randomly chosen one-second interval is plotted. The plot shows results for LIMERIC, $10 \mathrm{~Hz}$ with DCC and CAM with DCC approaches for the 1000 node case. The size of the time bins is 10 msec. For LIMERIC (Figure 6 (a)) and for $10 \mathrm{~Hz}$ with DCC (Figure 6 (b)); the message transmissions are more or less spaced uniformly in time. However, the transmissions for CAM with DCC approach appear in clusters. This clustering leads to many nodes transmitting at the same time resulting in a higher PER. And at other time instances, the wireless channel is hardly used. $10 \mathrm{~Hz}$ with DCC approach transmissions are much more uniformly spaced along time compared to CAM with DCC and this is why it has lower PER even when it has higher average CBR (Figure 13) as compared to CAM with DCC approach. This is consistent with the CBR plot in Figure 13 where CAM with DCC has higher variations than $10 \mathrm{~Hz}$ with DCC approach. In Figure 13, LIMERIC also has the smallest CBR oscillations and this leads LIMERIC and $10 \mathrm{~Hz}$ with DCC approach to have quite similar PER in Figure 4, even when LIMERIC converges at a higher average CBR.
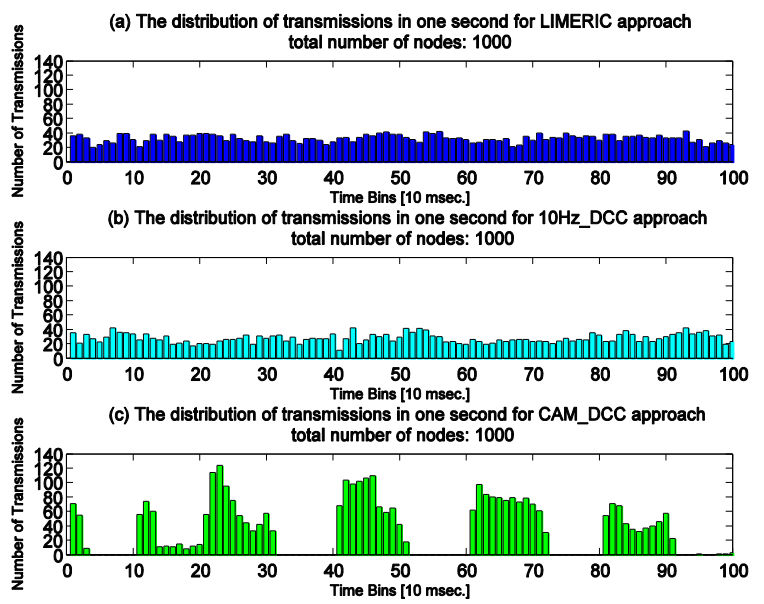

Figure 6. The distribution of the number of transmissions in a randomly chosen one-second interval for 1000 nodes for (a) LIMERIC, (b) $10 \mathrm{~Hz}$ with DCC and (c) CAM with DCC.

Further in Figure 5, the $10 \mathrm{~Hz}$ with DCC approach has higher PER than the LIMERIC approach. However, the two approaches has similar CBR values but since LIMERIC's CBR measurements are more stable, this leads to a lower PER for LIMERIC. It can also be observed in Figure 3 - Figure 5, that the fixed $10 \mathrm{~Hz}$ scheme does not control the channel load, hence its PER increases with the node density. For the 1500 node case (Figure 5) the fixed $10 \mathrm{~Hz}$ scheme has the highest PER.

In Figure 7 - Figure 9, the 95th percentile IPG for the different schemes for node densities of 500, 1000, and 1500, respectively, are depicted. In general, LIMERIC shows better performance than the other schemes. In Figure 7 and Figure 8, it is observed that for the first few distance bins, the 95th percentile IPG of fixed $10 \mathrm{~Hz}$ transmission approach is lower than LIMERIC.

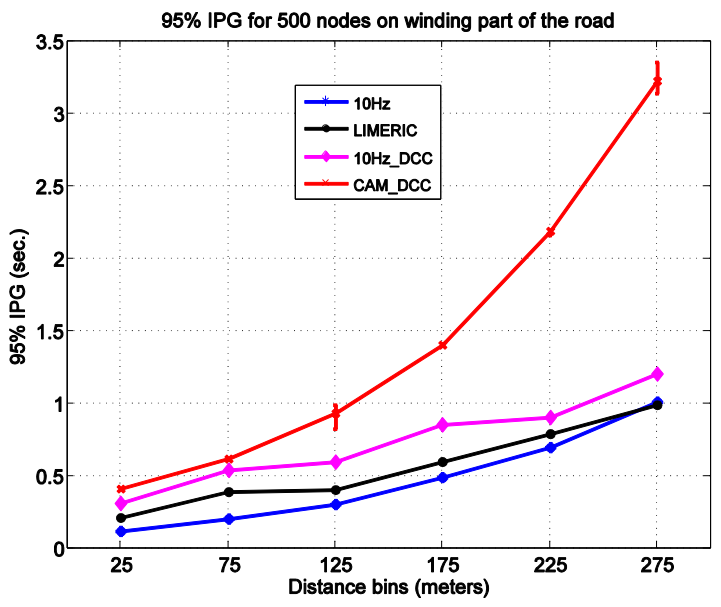

Figure 7. 95\% IPG for total number of nodes $=\mathbf{5 0 0}$.

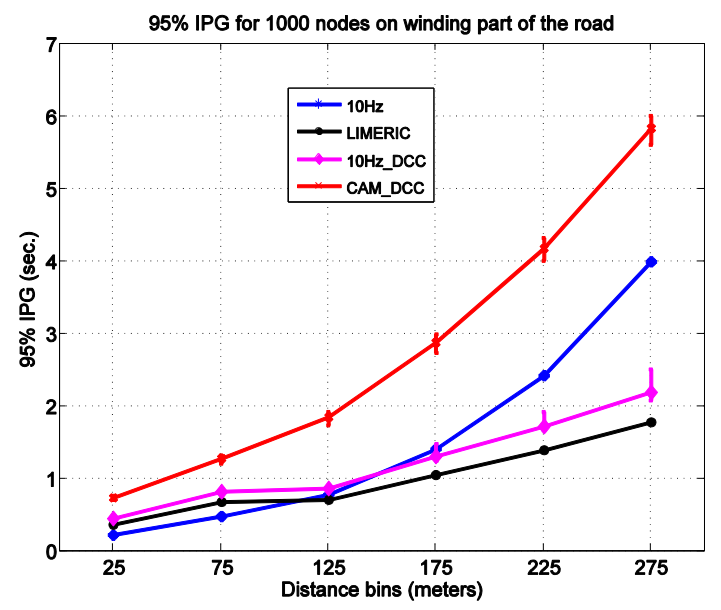

Figure $8.95 \%$ IPG for total number of nodes $=1000$.

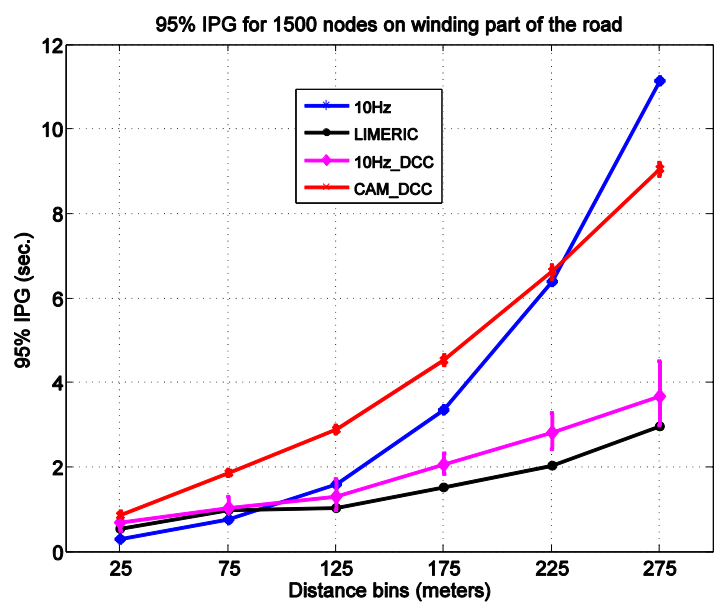

Figure 9. 95\% IPG for total number of nodes $=1500$.

This is because of that fixed $10 \mathrm{~Hz}$ transmits at a higher rate than LIMERIC and hence, has a better IPG performance 
even with a higher PER. However, for larger distances in Figure 8 and for a 1500 node density in Figure 9 the PER of fixed $10 \mathrm{~Hz}$ transmission approach becomes quite high and it has worse performance than LIMERIC, which adaptively controls the channel load. Similar to the PER results, CAM with DCC scheme has the worst performance due to the oscillations it causes in the CBR. We also observe that IPG performance of $10 \mathrm{~Hz}$ with DCC is worse than LIMERIC. This is because $10 \mathrm{~Hz}$ with DCC scheme does not optimize the use of the wireless channel and has a lower steady state message rate leading to higher IPG.

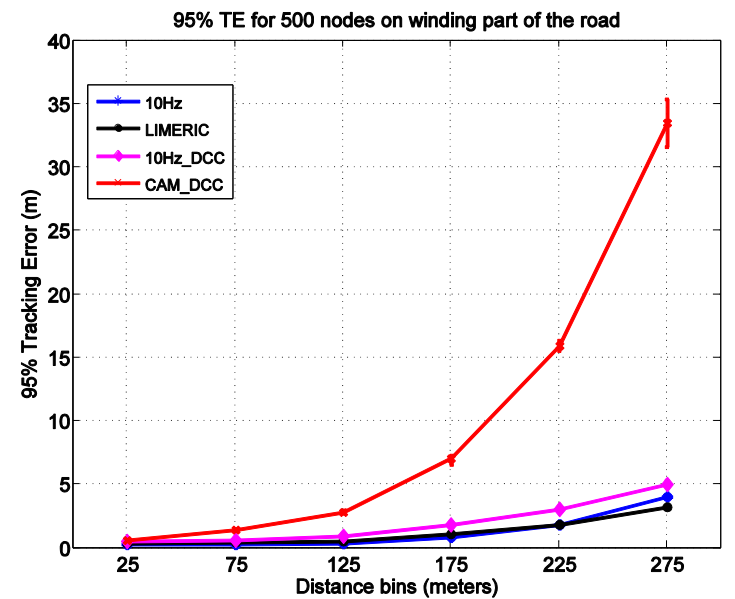

Figure 10. 95\%Tracking Error for total number of nodes $=500$.

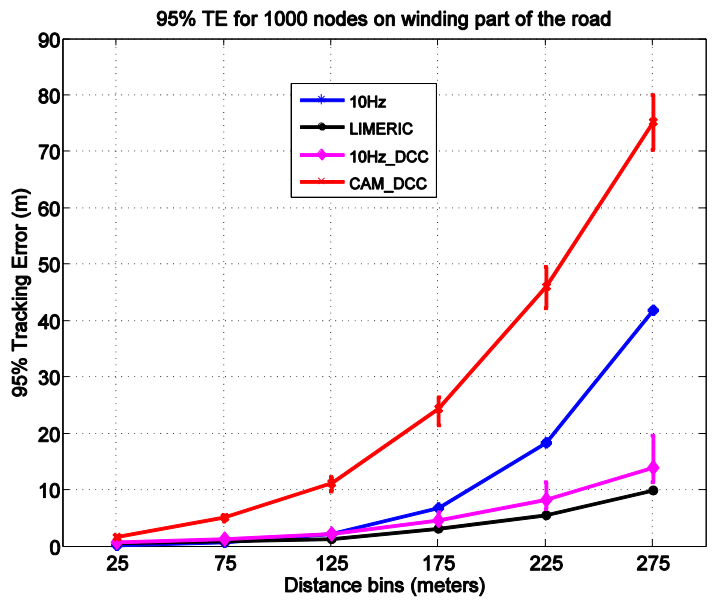

Figure 11. 95\% Tracking Error for total number of nodes $=1000$.

In Figure 10-Figure 12, the 95th percentile TE for all four schemes for 500, 1000, 1500 node density, respectively, are plotted. As in the IPG performance, LIMERIC outperforms all other schemes. Due to the CBR oscillations in CAM with DCC scheme, it generally has the worst TE performance. It can be noticed that fixed $10 \mathrm{~Hz}$ transmission has good performance at low node density (500 node case), but as node density increases the PER becomes high which also leads to higher TE performance. Also, $10 \mathrm{~Hz}$ with DCC scheme has a larger TE than LIMERIC. This is consistent with the IPG results.

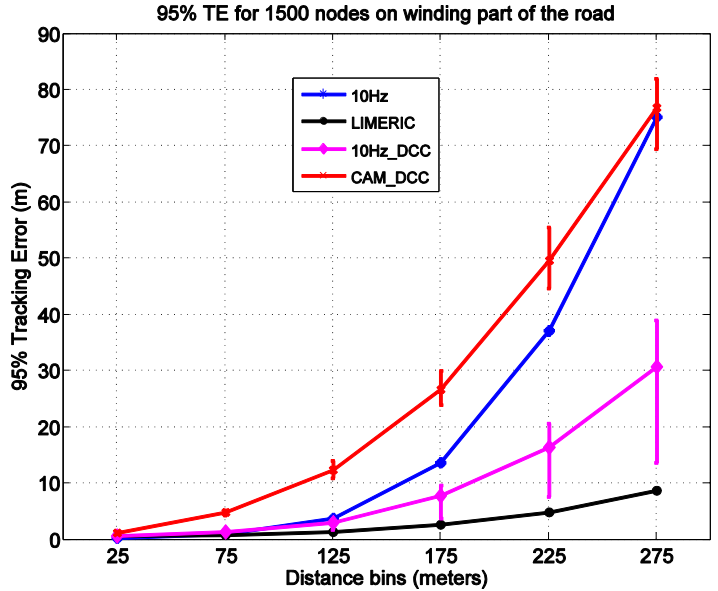

Figure 12. 95\% Tracking Error for total number of nodes $=1500$.

\section{Discussion}

In Figure 13, a CBR comparison between the four different schemes for the 1000 node case is plotted. LIMERIC shows that it can control the CBR target of 0.68 . The fixed $10 \mathrm{~Hz}$ scheme does not control the channel load and ends up at a very high CBR of 0.92. The CBR for CAM with DCC scheme has wide variation in two main levels, one with a range between 0.02 and 0.2 and the other with a range between 0.85 and 0.92 . The CBR for $10 \mathrm{~Hz}$ with DCC approach varies between 0.4 and 0.7 .

A possible cause for the high CBR variations for the CAM with DCC approach is that the CBR measurements are synchronized across all nodes. This leads to that all nodes measure a high CBR at the same time, leading to fewer CAM generations. The fewer CAM generations influence the CBR, which decreases during the coming CBR interval. The lower CBR value leads to higher allowed CAM generation rate that once again leads to higher CBR. This leads to two CBR levels as observed in Figure 13 for CAM with DCC approach.

In Figure 14, unsynchronized and synchronized CBR measurement intervals are depicted for the CAM with DCC approach. In the unsynchronized case, nodes have independent uniformly distributed measurement intervals. When the measurements are unsynchronized, the two "levels" of CBR values found for the synchronized case have disappeared, but the CBR value still varies in the range of 0.2 to 0.8 . Hence, unsynchronized CBR measurement intervals would likely only improve the performance of the CAM with DCC scheme slightly and we would still expect PER, IPG and TE values to be quite high as observed in Section IV.B.

The average message interval for various schemes for the 1000 node case is shown in Figure 15. The fixed $10 \mathrm{~Hz}$ scheme generates messages at an interval of $100 \mathrm{msec}$. For the LIMERIC approach, the message interval is calculated based on the current rate at the end of every monitoring period. As observed from the plot with a node density of 1000, the LIMERIC algorithm converges to a message generation interval of $300 \mathrm{msec}$. For the $10 \mathrm{~Hz}$ with DCC scheme and the CAM with DCC scheme, the message intervals are obtained at the end of every CBR measurement period. Based on the latest measured $\mathrm{CBR}$, the message interval is decided according to 
Table 1. The CBR variations in CAM with DCC approach lead to a variation in the message generation interval between 200 msec and $500 \mathrm{msec}$. The $10 \mathrm{~Hz}$ with DCC approach has less CBR variations and its average message generation interval varies in a range roughly between $360 \mathrm{msec}$ and $420 \mathrm{msec}$.



Figure 13. CBR sampled at the winding part of the road versus time for various approaches.
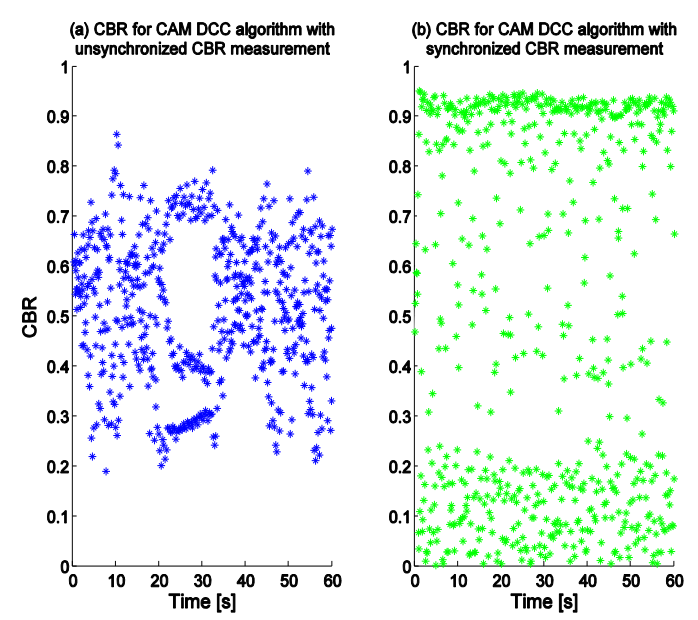

Figure 14. (a) the CBR for CAM with DCC approach with unsynchronized CBR measurement; (b) the CBR for the CAM with DCC approach with synchronized CBR measurement.

\section{CONCLUSIONS}

This paper compares two C-ITS/DSRC congestion control approaches: a reactive approach represented by the ETSI DCC framework, and an adaptive approach represented by the LIMERIC algorithm. We consider two DCC control points: access layer gatekeeping and facilities layer message generation. Using NS-2, we show that LIMERIC generally achieves lower reception intervals and tracking error than DCC. This is a result of LIMERIC's ability to drive channel load to a target chosen to achieve maximum throughput and awareness, independent of vehicle density. We also show that DCC is naturally oscillatory due to its discrete state definition, and furthermore that DCC can be truly unstable when it controls both gatekeeping and message generation. The DCC algorithm might be improved to reduce its oscillations and make its stability more robust. However, an improved DCC would still not have LIMERIC's convergence and steady state advantages; such a comparison merits further study.

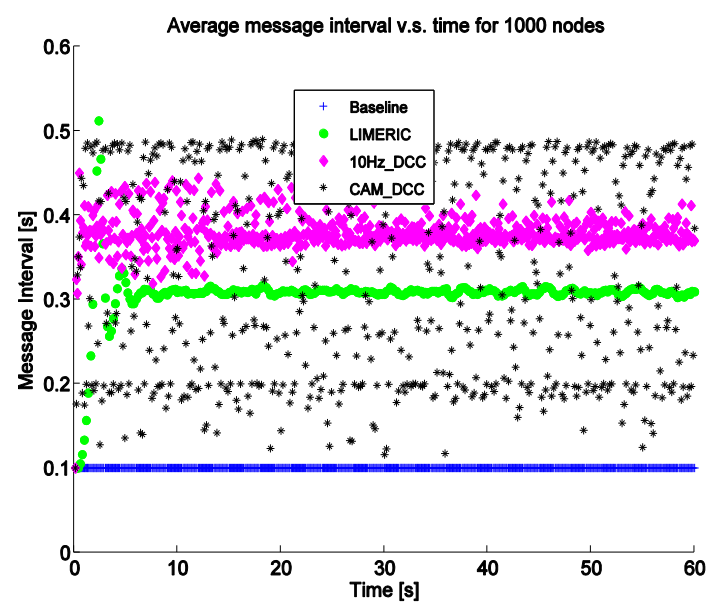

Figure 15. Averaged message generation interval for various approaches.

\section{REFERENCES}

[1] G. Bansal, J. Kenney, and C. Rohrs, "LIMERIC: A linear adaptive message rate algorithm for DSRC congestion control," IEEE Trans. Veh. Technol., vol. 62, no. 9, pp. 4182-4197, Nov. 2013.

[2] G. Bansal, H. Lu, J. Kenney, and C. Poellabauer, "EMBARC: Error model based adaptive rate control for vehicle-to-vehicle communications," Proc. 10th ACM VANET 201), June 2013, pp. 41-50.

[3] IEEE Standard for Information Technology - Telecommunications and information exchange between systems - Local and metropolitan area networks - Specific requirements; Part 11: Wireless LAN MAC and PHY specifications; Amendment 6: Wireless Access in Vehicular Environments, IEEE Std $802.11 \mathrm{p}^{\mathrm{TM}}$, July 2010.

[4] "Deployment Strategy for cooperative ITS in Europe," Car2Car Communication Consortium Memorandum of Understanding, June 2011

[5] "U.S. Department of Transportation Announces Decision to Move Forward with Vehicle-to-Vehicle Communication Technology for light Vehicles," NHTSA 05-14, Feb. 3, 2014.

[6] Intelligent Transport Systems (ITS); Vehicular Communications; Basic Set of Applications; Part 2: Specification of Cooperative Awareness Basic Service, ” Draft ETSI EN 302 687-2 v1.3.0, Aug. 2013.

[7] Dedicated Short Range Communications (DSRC) Message Set Dictionary; SAE Std J2735, SAE International, November 2009.

[8] ITS DCC Mechanisms for Intelligent Transport Systems operating in the 5 GHz range; Access layer part," ETSI TS 102687 v1.1.1, July 2011.

[9] ITS; Radio communications equipment operating in the $5855 \mathrm{MHz}$ to 5 $925 \mathrm{MHz}$ frequency band; Harmonized EN covering the essential requirements of article 3.2 of the R\&TTE Directive," ETSI EN 302571 v1.1.1, Sep. 2008. 